\title{
Effect of Sports Nutrition Supplement on the Increase of Physical Strength Based on Mobile Sensor Network Technology
}

\author{
Runping Zhang $\mathbb{D}^{1}$ and Xue Hong ${ }^{2}$ \\ ${ }^{1}$ College of Physical Education, Sanya University, Sanya, 572022 Hainan, China \\ ${ }^{2}$ Silk Road Business School, Sanya University, Sanya, 572022 Hainan, China \\ Correspondence should be addressed to Runping Zhang; runpingzhang@sanyau.edu.cn
}

Received 14 October 2021; Revised 30 October 2021; Accepted 6 November 2021; Published 23 November 2021

Academic Editor: Deepak Kumar Jain

Copyright (c) 2021 Runping Zhang and Xue Hong. This is an open access article distributed under the Creative Commons Attribution License, which permits unrestricted use, distribution, and reproduction in any medium, provided the original work is properly cited.

\begin{abstract}
With the development of signal processing technology and the appearance of small, low-cost, and low-power microprocessors, mobile sensor networks have begun to emerge in large numbers. In view of the different exercise abilities of different body components, mobile network sensor technology is used to monitor various data. Lean body mass is positively correlated with physical strength, aerobic capacity, and maximum oxygen uptake and is the main factor affecting explosive power, speed, and endurance. This article mainly studies the effect of nutritional supplementation in sports on physical strength. The subjects in this article are 10 male physical athletes. Three solid sports drinks with different nutrient composition ratios were selected from the national team's nutrition centralized procurement product list, and a placebo with sugar-free sweetener and purified water was used as a control group. The test measures the maximum oxygen uptake to determine the exercise intensity during the formal exercise test. Each stage includes exercise test day and washout period. Eat on the test day and provide a one-hour static rest after a unified breakfast, and then, perform a 1-hour power bicycle riding after the rest. During $0,15,30$, and 45 minutes of exercise, drink the designated solution at $1.8 \mathrm{~mL} / \mathrm{kg}$ body weight and record it as the corresponding solution group. Within 30 minutes immediately after the end of the cycling exercise, fluids should be refilled at $10 \mathrm{~mL} / \mathrm{kg}$ of body weight. Experimental data showed that the levels of lactic acid in each group increased significantly after exercise compared to those at rest $(P<0.05)$, and there was no difference between the groups. There was no significant difference in the duration of the exercise ability test between the sports drink group and the placebo group. The experiment in this article shows that athletes can take appropriate measures to improve athletes' physical activity level, increase daily energy consumption and a reasonable and balanced diet, which can effectively reduce the athlete's body fat percentage, improve, and maintain a reasonable body composition. Among all the monitoring actions, the monitoring results indicate that the evaluation value of the action of combat is the highest, reaching 8.7 .
\end{abstract}

\section{Introduction}

As one of the core technologies of the Internet of Things, mobile sensor networks have received more and more attention from researchers in recent years. Node location is a key function of many applications in mobile sensor networks, such as environmental monitoring, wild animal tracking, and medical patient monitoring applications, and location has become one of the most basic business functions [1]. A large number of sensors communicate with each other, process, and transmit information through a network of wire- less communication, which can monitor, perceive, and collect various environmental information or information about monitored objects in the network distribution area in real time and process and transmit this information to required users. Different body compositions have different effects on exercise performance. Lean body mass is positively correlated with physical strength, aerobic capacity, and maximum oxygen uptake. It is one of the main factors affecting explosive power, speed, and endurance. It is generally believed that absolute strength is directly proportional to lean body mass. Weight grading, heavy athletic events that 
focus on strength and explosiveness have high requirements for lean body mass; excess body fat in the body is considered a "dead" weight, which will affect a person's strength, speed, and endurance. The body fat percentage is significantly negatively correlated with the peak anaerobic power, and the anaerobic capacity of the two energy supply systems including phosphate and glycolysis is the energy basis for all shortterm high-intensity exercise. This shows that body composition has a close relationship with sports competition [2].

The body's long-term maintenance of a certain body weight is determined by the interaction of genetic characteristics and environmental factors. Therefore, weight loss is not a task that can be completed in a short time. When we decide to lose weight, we should carry out it slowly in combination with our own situation and realize weight loss through the long-term negative balance between calorie intake and calorie consumption. The main component of this method is fat. The effect of weight control requires the detection of changes in the athlete's body composition. This change in body composition can reflect whether the weight loss of the subject during weight control is a decrease in fat or a simultaneous loss of muscle. It can be timely prepared for simultaneous loss of muscle due to weight control, decreased muscle strength, and result in a decrease in athletic ability, and based on the body composition test results, you can understand the athlete's weight control potential and whether the weight control can achieve the expected result without losing physical fitness.

Sousa et al. analyzed the differences in sociodemographic and athletic characteristics, health behaviors, and food intake of athletes with and without nutritional supplements (NSs). They completed the NS usage questionnaire for high-performance Portuguese athletes from 13 sports, assessed sociodemographic information (gender, age, height, weight, and education level of athletes and parents), healthrelated information (smoking, (daily smoking time) sleep, walking, and sitting), and exercise (type, number of international performances, weekly training time, and weekly fitness time) characteristics and semiquantitative food frequency questionnaire for the first 12 months ( 86 items). They believe that original/value athletes who use NS have different characteristics from nonusers and seem to have healthier and more exercise-oriented food choices. Their findings may help sports and health professionals identify so-called or future NS users, so that they can develop timely, self-directed supplementary programs [3]. The use of nutritional supplements among athletes is very common. In this cross-sectional study, Wardenaar et al. assessed the prevalence of nutritional supplements used by a large number of Dutch competitive athletes in diet counseling. They believe that almost all athletes have used nutritional supplements during their sports career. Taking dietary counseling may lead to more informed choices in the use of nutritional supplements related to performance, recovery, and health. Their research is not targeted [4]. A series of nutrition education strategies can be used to help athletes improve their nutrition knowledge. The evaluation of nutrition education interventions can guide athletes to carry out effective nutrition education. Heaney et al. systematically review sports/general nutrition education interventions provided to athletes and evaluate their effectiveness. They searched using terms related to nutrition knowledge, athletes, education, and intervention. Research must be conducted among athletes (all calibers), using scored nutritional knowledge assessment tools, and measuring knowledge before and after nutritional education interventions includes peer-reviewed and unpublished papers. Most interventions in their study reported significant improvements in nutritional knowledge. However, due to the wide variety of knowledge assessment tools, limited verification, and the main use of face-to-face intervention, it is impossible to determine the most effective way and dose of nutrition education for athletes [5].

This research introduces the mobile sensor network technology in detail and discusses the architecture of the sensor, the composition of the mobile sensor network, and its characteristics and applications, from the composition of sensor nodes to the protocol framework of the sensor network, the topological structure of the mobile sensor network, the characteristics of the mobile sensor network and the related standards related to its performance, the application of the mobile sensor network in various related fields, etc.; based on reading domestic and foreign literature on the relationship between athletes' nutritional supplementation and physical strength gain, this study takes athletes as the main research object to observe the effects of sports training and nutritional interventions on athletes' physical exertion, so as to optimize sports training for athletes' health strategies, improving athletes' performance, etc., and provide important reference and application value.

\section{Physical Activity and Nutrition}

2.1. Physical Activity. Some experts and scholars mentioned in 2012 that ACSM will have five different types of physical activity issues: physical activity influencing factors and promotion of physical activity, survey of physical activity status, physical activity measurement tools, physical activity, and health. Among them, physical activity promotion and physical activity and health issues accounted for $72 \%$ of the total physical activity issues, reaching 36 , which proves that researchers are paying high attention to physical activity. At the same time, an objective and accurate method to measure the level of physical activity and health indicators is proposed, and a physical activity guide suitable for Chinese people is proposed. The in-depth study of the influencing factors of physical activity of different groups of people from multiple levels provides a practical basis for the design of physical activity intervention programs.

In the national physical fitness monitoring conducted nationwide in 2010, adult group physical activity has obvious regional differences in individual physical activity levels of Chinese residents; the number of people in the fully sitting and physically active classes in coastal areas is higher than in inland areas and in eastern areas in the western region. The accelerating pace of life in modern society and increasing life pressure have caused people to reduce physical activity and tend to various recreational activities to relieve stress. Epidemiological studies have found that lack 
of physical activity is one of the early causes of coronary atherosclerosis [6]. Regular physical activity can make people healthier, and for children, it can promote growth and mental health. For adults, it can reduce the incidence and mortality of stroke, diabetes, coronary heart disease, and other diseases. For the elderly, it can slow down the decline of muscle strength and the incidence of chronic diseases while maintaining good health. In the research on the impact of physical activity on cognitive ability, it is concluded that nonaerobic or aerobic exercises such as strength resistance and stretching exercises have a positive effect on cognitive ability. For the elderly, regular physical activity can maintain a certain level of cognitive ability to ensure the quality of life and prolong life. It is easier to judge physical activity as an intervention factor in research, but whether physical activity also plays a positive role in the acquisition of cognitive ability, it is necessary to conduct research on younger people, even starting from the stage of rapid development of cognitive ability in children for long-term follow-up research, so the relationship between physical activity and cognitive ability needs further research.

2.2. Nutrition. Nutrition studies the relationship between the body, diet, and health, as well as the effects and effects of the ingredients in these diets on human health. It is closely related to people's lives and plays an important role in disease prevention, protection, and improvement of health. In 2005 , experts in the international nutrition community announced the "Giesen Declaration" to promote new nutrition science, which gave a new definition of nutrition, that is, "the study of food systems, foods, and beverages and the nutrients and other ingredients contained in them, including their biological, social, and a discipline that interacts with environmental systems" [7].

There are broad categories of nutrition, and the research directions are carefully divided. According to the different research priorities, it can be divided into basic nutrition, public nutrition, maternal and child nutrition, geriatric nutrition, clinical nutrition, food nutrition, special population nutrition, and chronic disease control nutrition. The main research direction of this subject is food nutrition. Food nutrition, as the name suggests, is to study the relationship between food, nutrition, and the human body and to improve the nutritional value of food and promote the health of the body through food. It mainly describes the basic nutritional theory, the nutritional value and characteristics of different types of food, and the nutritional needs of different groups of people and provides guidance and help for people. In recent years, food nutrition has received extensive attention and attention, and more and more disease problems are involved in food nutrition. The knowledge in food nutrition has been widely used in clinical medicine practice, and various types of food nutritional ingredients, value, and influence will be more known and used by the public.

2.2.1. The Problems of Food Nutrition in People's Consciousness. Before the reform and opening up, my country's economic level was poor, food and clothing were not guaranteed, food was scarce, and the diet structure was simple, causing many people to be in poor health due to malnutrition. Today after the economic turnaround, abundant material conditions and a wide variety of food have caused a high incidence of diseases. People are often confused by this. The long-term lack of material is in sharp contrast with what you want to eat. People start to supplement and eat all kinds of foods. They think that eating more expensive and eating more is good for the body but ignore excessive food intake and nutrition. It accumulates in the body and causes harm to the body. Therefore, people should establish a correct understanding of food nutrition and apply it scientifically and reasonably in life.

\subsubsection{Problems of Food Nutrition in Publicity. The develop-} ment of economy and technology has promoted the improvement of material life. Food and nutrition has gradually become known in daily life. People can use different media to receive relevant information, such as books, magazines, newspapers, radio, television, interviews, and mobile phones. However, people's age, lifestyle needs, and other good and bad things are different. Although there are various publicity methods, there are still some problems. For example, in books and magazines, the amount of text reading is large, which is easy to make people visually fatigued, and it is difficult to quickly find effective information. Many people are not easy to understand. Secondly, in TV interviews, although they are time-sensitive and scientific, they are all experts in related fields and have high credibility. However, due to time constraints, answers have limitations. At the same time, TV, radio, interviews, and other publicity methods are all momentary memory is difficult for people to remember for a long time, and it is easy to miss and miss key information in such propaganda [8].

\subsubsection{Problems of Food Nutrition in the Publicity Content.} Nowadays, many people's understanding of food nutrition is actually one-sided. For example, people only know that fruits and vegetables contain a lot of vitamins and minerals, but they do not know that there are also a lot of sugars in fruits. After overeating, they cannot be fully utilized and absorbed by the body. It will lead to diseases; people know that soy products are good for health and are rich in protein, but they ignore the decline of kidney function and the formation of arteriosclerosis caused by excessive intake; they know that yogurt can supplement the probiotics needed by the human body but forget it can also increase blood lipids and blood sugar. All kinds of indications are that in the promotion of food content, we only focus on its benefits and good aspects, while ignoring the differences in human needs and its drawbacks.

2.3. Sports. Lack of purpose is the most common deviation of college students when they study physical education. What is the learning of physical education? What is the ultimate result? Most students have never thought about it. This psychology leads to physical education. Lack of concentration in class, just for class, and lack of interest and learning direction greatly reduce the enthusiasm for sports participation. 
Therefore, in teaching, teachers should pay attention to guiding students to set clear goals and know the purpose of physical education. Teachers should carry out more extracurricular activities and properly guide students' initiative. Of course, due to different genders, male and female students are different in learning. Teachers should consider as many factors as possible in each class [9]. Goals that are too high or too low will make students tired of learning. In the physical education class, you should choose moderately difficult teaching goals and let students understand their tasks. This is an effective means to stimulate the engine. Under the premise of proper goal orientation, goal setting is a strategy to motivate students to participate in sports. Goal orientation refers to the achievement goal orientation on which an individual participates in an activity. It is not the quantitative standard of behavior that is specifically required to be achieved, but the achievement orientation pursued in the hearts of students. In view of the poor situation of college students in physical education, I think our research will be more meaningful only if we properly stimulate the enthusiasm of students to actively contact sports and pay attention to the physical growth of sports nutrition supplement. For example, some students take physical education classes with the goal of mastering technology and improving physical fitness, aiming to develop their personal abilities, while some students are aiming at competing and surpassing others, expecting to express themselves and win honor, prestige, and status. The former is to master task orientation, and the latter is self-expression orientation. Together with the students' belief in sports ability, they have different influences on their motivation and behavior types to participate in sports activities. Sports workers should appropriately encourage students' self-expression behavior and at the same time guide them to improve their skills, develop their physical fitness, temper their will as the ultimate orientation of sports learning and exercise activities and strive to continuously improve their physical ability.

Achievement motivation is a higher-level social motivation, which refers to the psychological tendency of individuals to actively engage in activities that they consider important or valuable and strive to achieve perfection and achieve excellent results. It is generated on the basis of achievement needs and is the internal driving force learned in social interaction. The cultivation of sports achievement motivation is the individual's "consciousness" of selfbehavior related to sports learning achievement motivation; through games, competitions, or other related activity arrangements, individuals can realize the relationship between setting goals, adopting behavior strategies to achieve goals and success or failure, and the impact of success or failure on emotional experience, resulting in the "experientialization" of success and failure experience; through the teaching and understanding of concepts such as "sport goals," "mental orientation," and "success criteria" related to achievement motivation, these concepts are "conceptualized" in students' minds, by changing "norm reference" to "self-reference," that is, more emphasis on the comparison before and after exercise, so that they can get more "success opportunities"; let the individual learn success criteria and behavioral strategies are applied to a certain physical exercise content, and you choose your own activity goals, strategies, and evaluation criteria, and conduct selfanalysis and evaluation of motivation levels, behavioral performance, and emotional responses [10].

The stimulation of exercise motivation has long been the focus of the reform of physical education and teaching ideas. At present, the guiding outlines successively promulgated by the relevant national education departments also fully reflect the importance of the subjective emotional experience of students. Among them, the advocacy of happy sports, the active introduction of optional teaching, and the establishment and operation of various sports clubs provide a strong guarantee for the creation of a good teaching atmosphere and at the same time enrich the physical education model. These new teaching models fully embody the principle of student-oriented and student-oriented. Students learn in a learning environment where their subjective initiative is brought into play, so that students can truly experience the happiness that sports bring, thereby more effectively increasing their interest in sports learning. Literature research shows that interest is the main component of motivation, and interest has a positive effect on the induction of motivation, the choice of behavior, and the maintenance of behavior. A lot of physical education teaching practical experience has proved that the diversity of teaching methods, the novelty of teaching modes, the joy of classroom atmosphere, and the practicality of teaching content will effectively cultivate and stimulate students' interest in sports learning [11].

2.4. Mobile Sensor Network Technology. The mature development of various basic technologies makes mobile sensor network technology a hot frontier subject with highly integrated knowledge and complete structural system. Node location and time synchronization, as the two key parts of every self-organizing and location-aware wireless sensor network, have always been the key topics of research and application. Node location refers to the location of the node that exports, sends, or receives synchronization information in the mobile path. Time synchronization refers to adjusting the time synchronization of the mobile sensor by using the synchronization signal. At present, the existing research separates and considers the mobile path planning and the route from the common node to the sink node but does not comprehensively consider the relationship between the selection of the sink node, the route from the common node to the sink node, and the moving path. If the sensor node is exhausted because of energy if it fails to work, there will be new problems such as network topology changes and routing reestablishment and it may even cause interruption of network communication. Although node location and network time synchronization have been studied together for many years, most of the research results are obtained on the basis of certain assumptions. Therefore, solving the problem of node time location has always been a research hotspot. Solving the problem of node clock synchronization is also to deal with node location in various environments. With the development and popularization of the Internet of Things, mobile devices such as mobile phones will not 
only be an interface for people to access the network, but also an intelligent terminal that connects with the surrounding physical world. As a mobile node or mobile agent of wireless sensor networks, compared with general sensor network nodes, mobile devices can artificially control its energy and storage capacity without being constrained by transmission distance and have strong computing power. The structure of the sensor network technology system is shown in Figure 1.

The function of each part is different. The work of the sensor node is completed by mutual cooperation. The function of the sensor module is to monitor and collect all kinds of data in the service area in real time, such as sound, temperature, humidity, location, and communication, what type of specific collection the data is determined according to actual needs, and the digital-to-analog conversion of the signal is completed at the same time. Centralized positioning algorithm refers to the relatively high hardware configuration of the central node, which can receive various types of information sent by other nodes, run the positioning algorithm, and realize the positioning of the network node after analysis and calculation. The response transmission between the sending node and the receiving node through the signal that is as follows:

$$
\begin{aligned}
Q_{i}(s) & =\frac{1}{g_{0}} \int_{i}^{s_{0}} g_{0}(s) d s+\frac{Q_{i}\left(s_{0}\right)}{x_{1}\left(s-s_{0}\right)}+y_{1}, \\
s & =\frac{1}{x_{i}} Q_{i}(s)+\frac{\left(s_{0}-y_{1} / x_{1}\right)}{Q_{i}(s)} .
\end{aligned}
$$

In terms of calculation, you must consider the time $g_{0}$ and the sequence $x_{1}, y_{1}$ of the event. When processing node information, you must also consider the problem of time. If there is an error $\mathrm{d}$ in the time, the synchronization cannot be maintained, and the information $s_{0}$ of different nodes cannot be merged. Define this process, and there are

$$
\begin{gathered}
Q_{j}(s)=\frac{x_{j}}{x_{i}} Q(s)+y_{j}-\frac{x_{j}}{x_{i}} y_{i}, \\
S_{i, l}\left(h S+\delta_{l}\right)=\bar{S}_{i, l}\left(h S+\delta_{l}\right)+m_{i}\left(h S+\delta_{l}\right) .
\end{gathered}
$$

The distance difference $Q_{j}(s)$ can be calculated by only using the node communication to transmit the communication signal of the physical layer $l$, and the phase difference of the sending node signal can be measured by using multiple receivers $\delta_{l}$ or antenna arrays to obtain the angle information of the sending node relative to the receiving node $h$. This process can be expressed as follows:

$$
\begin{gathered}
\beta_{i j}\left(h S+\delta_{l}\right)=\varphi_{i j}\left(h S+\delta_{l}\right)+m_{i}\left(h S+\delta_{l}\right)-m_{j}\left(h S+\delta_{l}\right), \\
s_{-l, t}(h)=\bar{s}_{i, l}\left(h S+\delta_{l}\right)-d_{i l}-\frac{\left[\bar{s}_{i, l}\left(h S+\delta_{l}\right)-d_{i t}\right]}{1+\alpha_{i}} .
\end{gathered}
$$

In practical applications, in the $m$-th cycle of sensor node configuration monitoring $\beta_{i j}$, the transmission delay of the signal error value $m_{i}, m_{j}$ between the node signals is established by the ideal value of $d_{i l}$, and this value is approximated according to the relative frequency offset. Expressed by the formula as

$$
\begin{aligned}
\varphi_{i j}\left(h S+\delta_{l}\right) \approx \varphi_{i j}\left(h S+\delta_{l}\right)+\varepsilon_{i j}\left\|s_{j, l}\left(h S+\delta_{l}\right)-s_{j, t}\left(h S+\delta_{l}\right)\right\|, \\
\varphi_{i j}\left\|(h+1) S+\delta_{l}\right\|=\varphi_{i j}\left(h S+\delta_{l}\right)+\varepsilon_{i j} \\
\cdot \cdot\left\{\left[s_{j, l}(h+1) S+\delta_{l}\right]-(h+1) S+\delta_{l}\right\} .
\end{aligned}
$$

Assumption $\delta_{l}=0$, and the above formula can be simplified to the following:

$$
\begin{aligned}
\varphi_{i j}[(h+1) & =\varphi_{i j}(h)+\varepsilon_{i j} S_{j}(h+1, h), \\
\varepsilon_{i 1}(h+n) & =\varphi_{i 1}(h+n)+m_{i}(h+n)-m_{1}(h+n) .
\end{aligned}
$$

Among them, $n=1, \cdots, M-1$, and $\varepsilon_{i 1}(h+n)$ and $\varphi_{i 1}(h$ $+n)$ are two adjacent nodes, and the relationship between the data measured by the sensor and the real data can be expressed as follows:

$$
\begin{aligned}
\varphi_{i 1}(h+n) & =\varphi_{i 1}(h+M-1)-\varepsilon_{i 1} S_{1}(h+M-1, h+n), \\
i(h+n) & =I_{M-1} \otimes[1,-S(h+M-1, h+n)] .
\end{aligned}
$$

Equation (11) converts this relationship into a matrix form for calculation. In the system, the sensor is an independent recording instrument and uses covariance equalization to record various data as marker signal $M-1$. When it ends, synchronous compensation is performed. The original data follows the cycle is updated at the beginning, and the formula is calculated as follows:

$$
\begin{aligned}
& W_{x y}\left(h S+\delta_{0}\right)=S_{I, 0}\left(h S+\delta_{0}\right)-s_{J, 0}\left(h S+\delta_{0}\right), \\
& W_{i j}\left(h S+\delta_{0}\right)=\varphi_{i j}\left(h S+\delta_{0}\right)+l_{i 0}-l_{j 0}+m_{i}\left(h S+\delta_{0}\right)-m_{j}\left(h S+\delta_{0}\right),
\end{aligned}
$$

$$
l(x, y)=\frac{1}{(2 \pi)^{m-1 / 2}} Q_{i}-\frac{g(l)}{(m)} .
$$

Through the above method, the iterative process can be divided into two steps to solve this problem. The first is to divide multiple grids to search $W_{x y}\left(h S+\delta_{0}\right)$, and the second is to iterate the search result as the initial value to obtain the global minimum $l(x, y)$, using the least square method is calculated as follows:

$$
\begin{aligned}
\bar{\gamma}_{i}^{2} & =\frac{1}{m-1}\left[\min \eta_{i j}-l_{i j}\right]=\frac{1}{M-1} \sum_{m-1}^{n-1} \min \eta_{m n}, \\
\bar{\eta}_{i l}(h S) & =1 / 2\left[\bar{s}_{l, i}\left(h S+\delta_{i}\right)-s_{l, l}\left(h \bar{S}+\delta_{l}\right)\right] \varepsilon_{i} .
\end{aligned}
$$

Unbiased value $\bar{\eta}_{i l}$ in the positioning model, the minimum deviation rate of the node transmission delay is min 


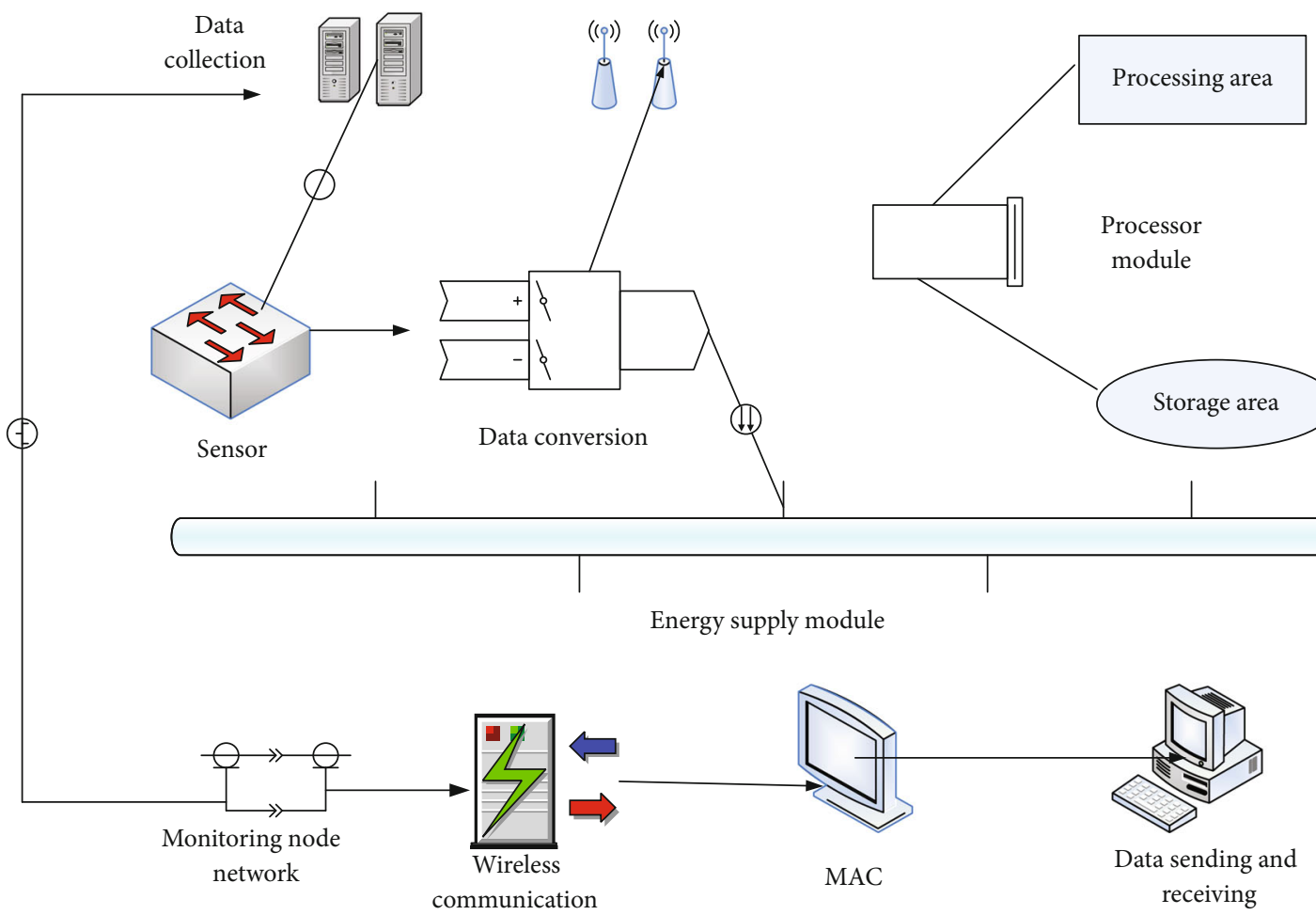

FIgURE 1: System structure of sensor network technology.

$\eta_{i l}$, and its authenticity is represented by the frequency difference $\bar{\gamma}_{i}^{2}$. The optimal distance point in the process of sensor data processing is calculated, and then, there is

$$
\begin{aligned}
D_{i j} & =\frac{1}{2}\left[s_{i j}\left(h S+\delta_{j}\right)-s_{i j}\left(h S+\delta_{i}\right)\right], i>j, \\
\bar{\gamma}_{d} & =\frac{1}{2 \pi} \min \left[\left(\varepsilon-g\left(\gamma_{d}\right)\right)^{-i} W_{d}\left(\varepsilon-g\left(\gamma_{d}\right)\right)_{d}^{-1}\right], \\
g\left(\bar{\gamma}_{d}\right) & \approx g\left[\bar{\gamma}_{d}(n)\right]+w\left[\bar{\gamma}_{d}(n)\right] \forall_{d}(n) .
\end{aligned}
$$

$g\left(\bar{\gamma}_{d}\right)$ is the result of $n$ iterations, forming a continuous linear process. Calculate $D_{i j}$ to avoid linear errors.

\section{Experimental Design on the Effect of Nutritional Supplementation on Physical Strength}

3.1. Experimental Design. Ten male football second-level athletes from sports universities are selected as the research objects. In order to rule out the effect of taking creatine supplements on skeletal muscle creatine levels before the experiment, all the subjects had not taken protein, amino acids, or creatine nutrition products within 5 weeks before the experiment. The subjects signed an informed consent form before the experiment and were randomly divided into the musclebuilding powder+glutamine group (group A), the musclebuilding powder group (group B), and the placebo group (group C). During the experiment, the number and intensity of regular training were maintained, and the diet was unified according to the requirements throughout the experiment period. There were no behaviors such as drinking, caffeine intake, and staying up late [12]. As shown in Table 1, it is the general information of the research object.

Acute resistance exercise. Measure the subject's maximum strength (1RM) for squatting and bench press before each formal test, and perform the following test according to the test value: squat $70 \% 1 \mathrm{RM}, 4$ groups, $6-8$ per group times, rest 60 seconds between groups, 90 seconds after the completion of the fourth group; then, perform 4 groups of $50 \% 1 \mathrm{RM}$ squat exercises, each group 6-8 times, rest between groups for 60 seconds, rest 150 seconds after the completion of the fourth group; bench press $70 \% 1 \mathrm{RM}, 4$ groups 6-8 times in each group, $60 \mathrm{~s}$ rest between groups, $90 \mathrm{~s}$ rest after completion of the 4 th group; and finally 4 groups of 50\% 1RM bench press exercises, 6-8 times per group, $60 \mathrm{~s}$ rest between groups, acute. The total exercise time of resistance training is about 30 minutes. The subjects were required to complete the joint motion to the full range each time. Acute endurance exercise: this article uses a onetime incremental load pedaling power bike experiment as a reference; set the initial load to $60 \mathrm{~W}$, increase by $25 \mathrm{~W}$ every 3 minutes, and maintain the speed at $60 \pm 2$ times/min, until the subject is unable to persist in completing the corresponding load movement.

3.2. Test Environment. The central air conditioner controls the indoor temperature and humidity and uses a thermometer and hygrometer to record the temperature and relative humidity every 15 minutes. During the whole experiment, the temperature range is $23 \sim 25^{\circ} \mathrm{C}$, the average value is 
TABLE 1: General information statistics of research objects.

\begin{tabular}{lcccc}
\hline Group & Age & Height $(\mathrm{cm})$ & Weight $(\mathrm{kg})$ & Training years \\
\hline A $(n=S)$ & $21.6 \pm 0.48$ & $176.9 \pm 3.46$ & $69.8 \pm 3.79$ & $5.3 \pm 1.32$ \\
B $(n=S)$ & $21.7 \pm 0.57$ & $177.2 \pm 3.37$ & $68.4 \pm 5.52$ & $6.5 \pm 3.78$ \\
C $(n=S)$ & $22.4 \pm 0.52$ & $175.2 \pm 3.84$ & $68.9 \pm 6.81$ & $4.5 \pm 2.14$ \\
\hline
\end{tabular}

$25.08 \pm 0.64^{\circ} \mathrm{C}$, the relative humidity range is $40 \% \sim 65 \%$, the average value is $56.96 \pm 5.29 \%$, the indoor air pressure range is $998 \sim 1010 \mathrm{mbar}$, and the average value is $1004.11 \pm 5.16$ mbar.

3.3. Test Indicators. Osmotic pressure. The freezing point method is used to determine the osmotic pressure of plasma and urine. Freezing point osmotic pressure tester, use a micropipette (VistaLabTM Technologies, Mt. Kisco, NY10549, USA) to add samples. Read the measured osmotic pressure through the display. The average value of the three measurements is the final osmotic pressure value. Total protein: $1 \mathrm{~mL}$ is drawn from the prepared plasma, and the colorimetric method is used to determine the total plasma protein content with an automatic biochemical analyzer (Beckman AU680). The reading shows the total plasma protein. $\mathrm{Na}+$, $\mathrm{K}+$, and $\mathrm{Cl}$ - are the three ions in blood and urine samples, using the same automatic biochemical analyzer, and each sample is tested three times, and the average of the readings is the final ion concentration. Blood lactic acid: from the collected EDTA anticoagulated vein, take $20 \mu \mathrm{L}$ of whole blood from the blood, add it to the preprepared $40 \mu \mathrm{L}$ rupture fluid diluent, shake enough to complete the rupture of the membrane, and use the YSI 1500 lactic acid meter to measure the venous blood lactic acid; the measurement method used by the instrument is lactate dehydrogenation enzymatic method. The content of lactate dehydrogenase Enzymatic $(\mathrm{LDH})$ is very rich in the cytoplasm. Under normal conditions, the cell cannot pass through the cell membrane, but it can be released outside the cell when the cell is damaged or dead. At this time, the activity of LDH in the cell culture medium is directly proportional to the number of cell death. The LDH activity is measured by colorimetry and compared with the LDH activity of the control hole of the target cell, and the wound killing fraction of effector cells to target cells can be calculated. The experimental method is simple and fast.

3.4. Mathematical Statistics. The data obtained in this study were sorted and analyzed, and the spss19.0 software and Excel statistical software were used for data processing. Use data as evidence, conduct scientific analysis, put forward opinions, and draw conclusions.

\section{Analysis of the Effect of Nutritional Supplementation on Physical Strength}

4.1. Application of Mobile Sensor Network in Motion Monitoring. The mobile sensor network digitizes and informatizes the objective physical world, so that humans can further understand and perceive nature. Citing the concept
TABLE 2: Sink node and sensor node.

\begin{tabular}{lcc}
\hline Parameter & Sink node & Sensor node \\
\hline Calculate ability & $>110$ & $<10.0$ \\
Storage capacity & $>60 \mathrm{M}$ & $<9.5 \mathrm{~K}$ \\
Power supply & Mains power supply & Battery powered \\
\hline
\end{tabular}

TABle 3: Node list related data.

\begin{tabular}{lccc}
\hline & $\begin{array}{c}\text { Node short } \\
\text { address }\end{array}$ & $\begin{array}{c}\text { Packet } \\
\text { length }\end{array}$ & $\begin{array}{c}\text { Bandwidth } \\
\text { requirement }\end{array}$ \\
\hline Work node & 1 & 50 & 1.07 \\
list & 3 & 80 & 2.19 \\
Free node & 2 & 50 & 1.11 \\
list & 4 & 100 & 2.37 \\
\hline
\end{tabular}

of "Smart Earth" put forward by US President Obama in 2009, the tasks of wireless sensor networks are as follows: more thorough perception, wider interconnection, and deeper intelligence. Its appearance has aroused widespread concern all over the world, and it is recognized as one of the technologies that will have a huge impact on the 21 st century. With the advancement of science and technology, the functions of nodes have become more and more powerful, and the network model in the LEACH protocol can be regarded as an abstraction of a distributed information collection system. It has incomparable advantages over other networks in terms of information acquisition and can be monitored in real time. And collect the information of various monitoring objects in the network distribution area. The main parameters of the sink node and the sensor node are shown in Table 2.

In the traditional mobile sensor network mobile handover scheme, the mobile node may fail the handover for some reason. The general approach is to directly disconnect from the network and restart the whole handover process. This will not only increase network energy consumption and generate frequent signaling interactions but also interrupt the provided services, especially bringing intolerable consequences for services with high real-time requirements. The relevant data of the two types of work node lists investigated are shown in Table 3. The difference between work node list and free node list is that free nodes can provide fast processing for real-time and demanding services to help work nodes switch.

Mankind has entered a brand-new information age. The influence of knowledge economy and network informatization has changed people's lifestyles. Some special industries have applied wireless sensor network technology, which has made the technological content and speed reach unprecedented heights. Investigate the role of mobile sensor network technology in sports nutrition monitoring, as shown in Figure 2.

The positioning of mobile nodes is also a technical difficulty in wireless sensor networks. Although the traditional fixed node positioning method has achieved good research 


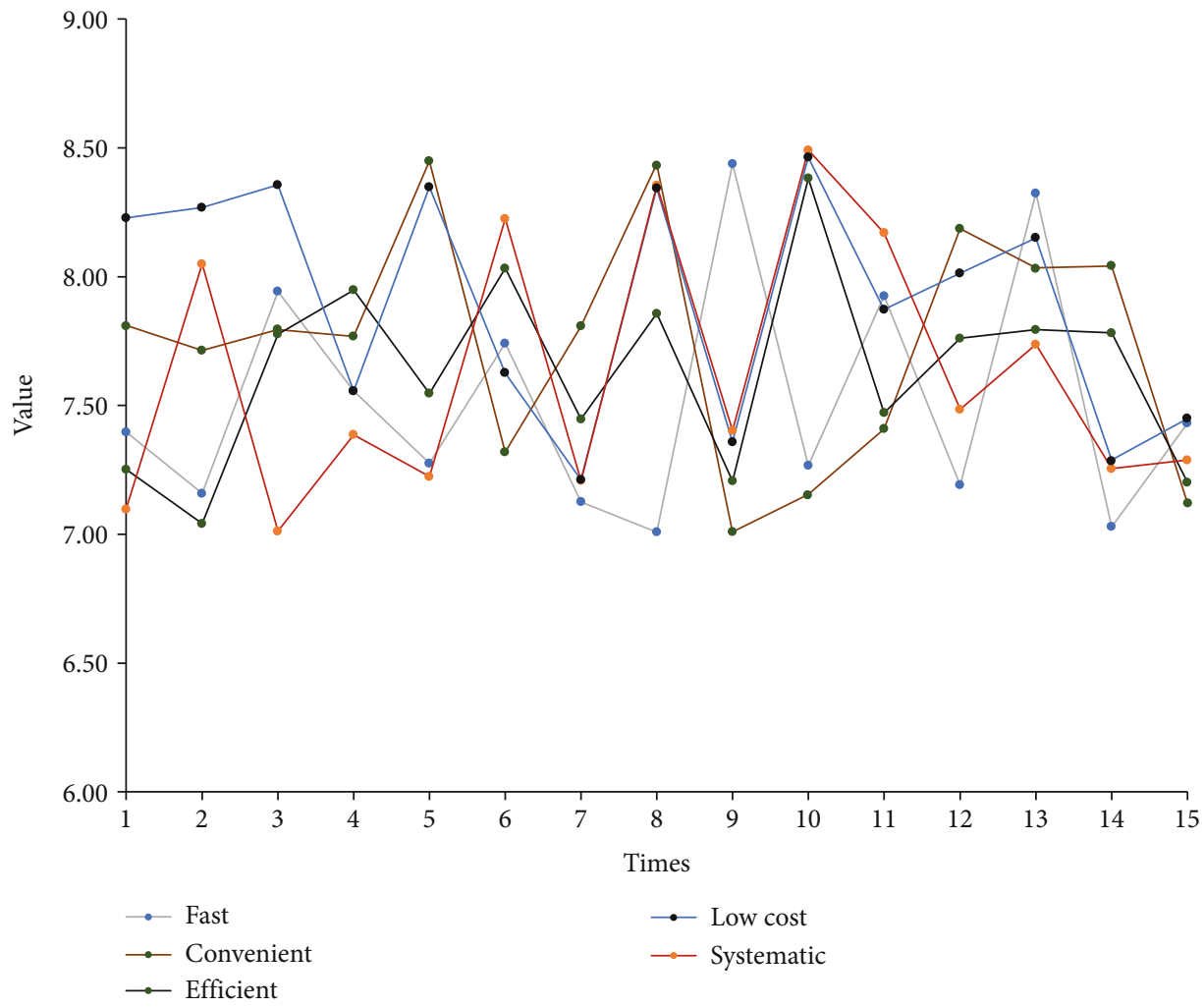

Figure 2: The effect of mobile sensor network technology on sports nutrition monitoring.

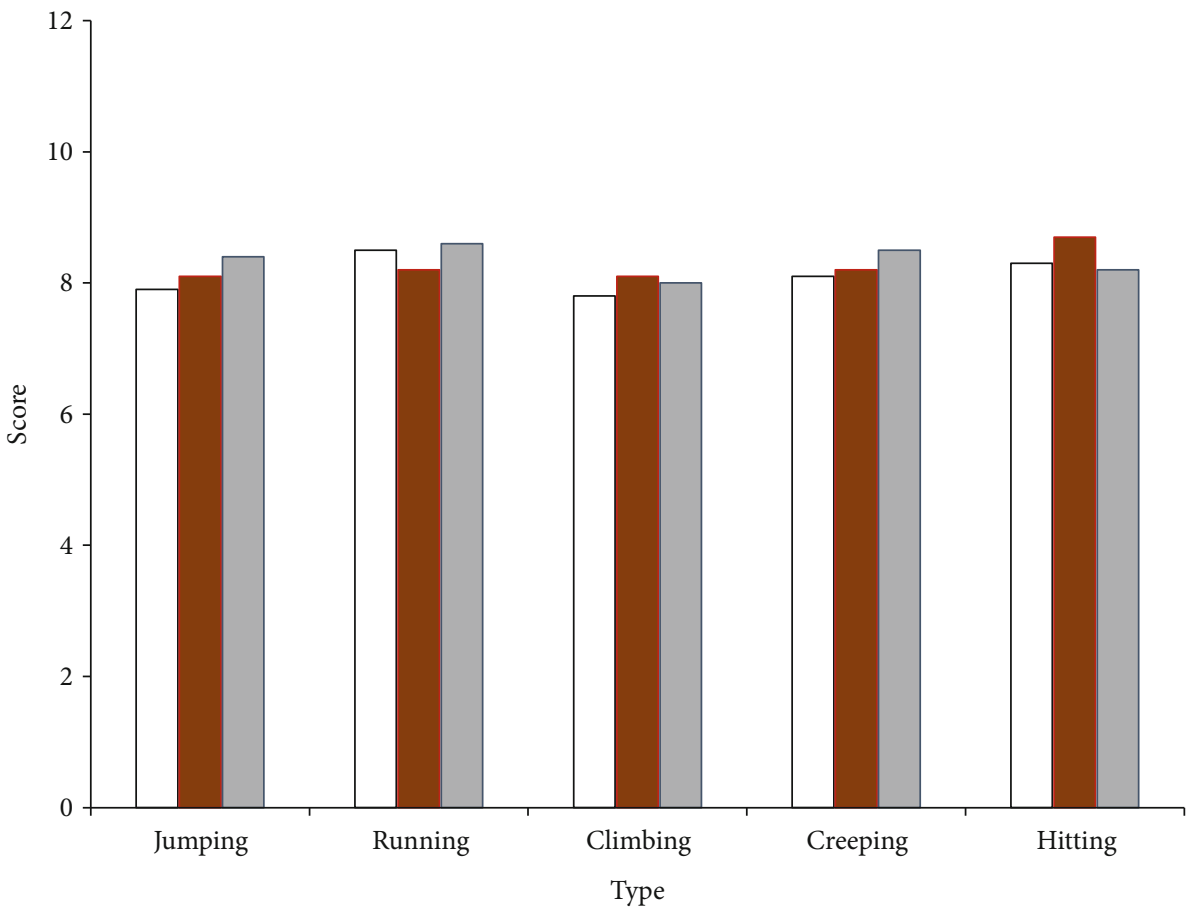

$\square$ Monitoring data

Monitoring results

Monitoring process

Figure 3: Mobile sensor network technology monitoring and evaluation of different actions. 
TABLE 4: Variance analysis of exercise frequency and exercise motivation.

\begin{tabular}{lcccrr}
\hline & 3 hours & 2 times a week & Once a week & $2-3$ times a month & $F$ \\
\hline Exercise motivation & $122.54 \pm 14.59$ & $101.57 \pm 14.57$ & $97.38 \pm 17.78$ & $92.34 \pm 7.65$ & 12.761 \\
\hline
\end{tabular}

results, it cannot meet the real-time requirements for mobile nodes. According to the needs in different scenarios, the types of data to be monitored will be different, and the network deployment methods will also vary according to the specific scenarios. For example, for an indoor environment, where the space area is very limited, you need to deploy fewer sensor nodes to complete the collection of indoor data. For different actions, the data processing effect that mobile sensor network technology can achieve in monitoring is shown in Figure 3.

From the data in the above figure, it can be seen that the evaluation value of the results obtained by using the mobile difference UN uplift network for monitoring under different actions is different. Among all the monitoring actions, the monitoring results indicate that the evaluation value of the action of combat is the highest, reaching 8.7.

4.2. Analysis of the Relationship between Exercise Frequency and Exercise Motivation. Athletes' training needs to be carried out regularly, including regular diet to ensure energy intake. Before the game, the main thing is not to break the dietary rules and establish good living habits. The most important thing is to maintain a normal rhythm of life. Eat more coarse grains rich in vitamin $\mathrm{B}$, peanuts and walnuts, fresh fruits, and vegetables rich in vitamin $\mathrm{E}$ to ensure normal and reasonable meal. Use reasonable physical and psychological adjustments to improve athletes' self-confidence, eliminate unhealthy tensions, and improve sleep quality, which is very important for restoring physical and mental strength.

Sports motivation refers to the internal motivation that affects individuals in sports. According to the source, it is divided into internal motivation and external motivation. It refers to the motivation from one's own active, curious, or competitive psychology, eager to obtain physical pleasure and psychological pleasure, stimulation, or personality development from sports activities. According to the direction, it can be divided into direct motivation and indirect motivation. The former points to sports learning content, method, or organization form, that is, it directly points to sports activities themselves. The latter points to the possible physical, psychological, and social results of sports activities. Table 4 shows the relationship between exercise frequency and exercise motivation.

As shown in Table 4, students who participated in 2-3 exercises a month scored the lowest, and students who participated more than 3 times a week scored the highest. There was little difference in motivation scores for students who participated in exercise twice a week and once a week. Afterwards, multiple comparisons showed that there were significant differences in the motivation of the participants who exercised 3 times a week, 2 times a week, 1 time, and 2-3 times a month $(P<03.01)$. It can be seen that as the frequency of exercise increases, the intensity of the students'
TABLE 5: Actual intake.

\begin{tabular}{lcccc}
\hline \multirow{2}{*}{ Number } & \multirow{2}{*}{ Total heat (kcal/d) } & \multicolumn{3}{c}{ Percentage of energy supply } \\
& & Sugar & Protein & Fat \\
\hline 1 & 3085 & 50.3 & 26.3 & 23.4 \\
2 & 2652 & 48.5 & 26.5 & 25.0 \\
3 & 2725 & 47.4 & 28.7 & 23.9 \\
4 & 2589 & 48.8 & 27.4 & 23.8 \\
5 & 2642 & 49.3 & 28.8 & 21.9 \\
\hline
\end{tabular}

exercise motivation becomes higher, reaching a very significant difference $(f=12.761, P<0.01)$.

4.3. Dietary Survey Results and Analysis. As shown in Table 5 and Figure 4, for athletes, the intake of protein and fat is generally high, and the intake of sugar is low. The carbohydrate intake is insufficient: the main reason is that athletes' meals take the form of buffets, and athletes generally believe that the more meat they consume in the diet, the more nutritious they are. On the contrary, staple foods such as rice, noodles, and some fresh foods containing more than $70 \%$ carbohydrates are almost completely ignored [13]. The caloric energy provided by carbohydrates in the diet of Chinese athletes is only $32-52 \%$ of the total caloric energy (a reasonable ratio is more than $60 \%$ ). Carbohydrates are the most ideal energy source for athletes' training and competition. Athletes with severely insufficient carbohydrate intake will not only seriously affect their training quality and athletic ability but also affect the normal metabolism of other substances.

As shown in Table 6 , the ratio of protein and fat intake is too high: excessive intake of fat and protein is harmful to exercise performance. The protein intake of Chinese athletes is $26-28 \%$ of the total calories (a reasonable ratio of $12-15 \%$ ), and the fat energy supply ratio is $22-23 \%$ (a reasonable ratio of $14-20 \%)$.

Heat energy is an important substance that constitutes the body. Athletes should pay more attention to the intake of heat energy and the three major substances during adolescence. Athletes must first adjust their food choices. Athletes must first adjust their food choices and choose more fruits, vegetables, and whole grains, while eating less and high. Fatty fast food, nutrition: the survey shows that in the diet of young athletes, the intake of protein and carbohydrates is insufficient, and the intake of fat is too high. The ratio of protein, carbohydrate, and fat to total energy in endurance young athletes' diet should be $12 \%-15 \%, 55 \%-60 \%$, and $25 \%-30 \%$, respectively. In addition, for athletes, one of the often overlooked ingredients in the diet is dietary fiber (cellulose). Half of the young people's daily intake of dietary fiber does not reach the minimum safe range. Foods rich in dietary fiber include fruits, vegetables, and whole grains. Since dietary fiber can retain more water in the colon, when 


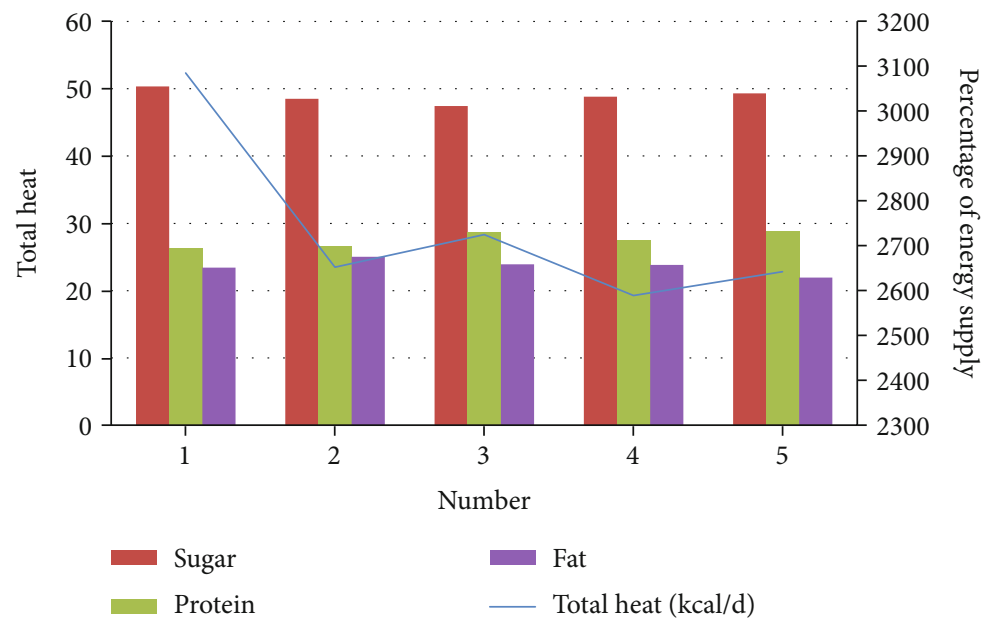

Figure 4: Actual intake.

TABLE 6: Recommended intake.

\begin{tabular}{lcccc}
\hline \multirow{2}{*}{ Number } & \multirow{2}{*}{ Total heat $(\mathrm{kcal} / \mathrm{d})$} & \multicolumn{3}{c}{ Percentage of energy supply } \\
& & Sugar & Protein & Fat \\
\hline 1 & 3590 & $60-65$ & $16-20$ & $13-17$ \\
2 & 3100 & $55-60$ & $18-22$ & $17-22$ \\
3 & 2980 & $60-65$ & $20-25$ & $13-17$ \\
4 & 2870 & $60-65$ & $20-25$ & $13-17$ \\
5 & 2830 & $60-65$ & $20-25$ & $12-15$ \\
\hline
\end{tabular}

eating fiber food, you should increase the intake of water and fruits. Athletes need to have enough confidence in eating "ordinary food" to promote muscle growth and improve athletic ability [14].

This paper takes 10 male athletes as the research object, selects three solid sports drinks with different nutritional components from the list of centralized nutritional procurement products of the national team, and uses placebo containing sugar-free sweetener and purified water as the control group. The test results are as follows.

As shown in Figure 5, it shows that the average 12minute running performance of the athletes in the experimental group increased from 2934.43 meters in the first week to 3132.42 meters in the second week, decreased to 3011.35 meters in the third week, and increased to 3146.28 meters in the fourth week. The average score of the control group in 12 minutes increased from 2,977.33 meters in the first week to 2,994.21 meters in the second week and dropped to 2,913.25 meters in the third week and increased to 3,052.39 meters in the fourth week. Compared with the first week, the results of the experimental group in the second and fourth weeks of the 12-minute run were significantly different (the paired $t$-test values were 2.96 and 3.21 , $P<0.05$, and the difference was significant). The results of the control group in the second and fourth weeks of 12minute running were not significantly different from those in the first week (the paired $t$-test values were 2.01 and 2.28, $P>0.05$, no significant difference). The results of the third week have declined relative to the second week.
As shown in Figure 6, it shows that the average performance of the 400-meter run of the experimental group increased from 69.76 seconds in the first week to $67.13 \mathrm{sec}$ onds in the fourth week after one month of phased training, while the average performance of the 400-meter run in the control group increased from 68.44 seconds in the first week to 68.46 seconds in the fourth week. The results of the experimental group and the control group are not significantly improved, but the results of the third week of the experimental group are significantly lower than those of the first week (the paired $t$-test value is $2.74, P<0.05$, and the difference is significant).

4.4. Effect of Supplementary Beverages on Acid-Base Balance. The analysis of the test results shows that there are three significant changes in the average performance of the experimental group and the control group in the 12-minute running event. The average performance of the experimental group in the second week of the 12-minute running event has increased significantly compared to the first week (relative to the first week). Run an extra 189 meters, and the third week was significantly lower than the second week (114 meters less than the second week), and the fourth week rose rapidly relative to the third week (136 meters more than the third week). The entire training performance is on the rise. In the control group, the average performance of the 12minute running in the second week did not rise significantly compared to the first week and was relatively stable. The third week was significantly lower than the second week (running 93 meters less than the second week), and the fourth week was relative to the third week. It rose rapidly again (running 146 meters more than in the third week). The overall training results showed little change in the first two weeks, and there were certain fluctuations in the second two weeks, and the overall situation was rising. The average performance of 400 runs in the experimental group during the entire training phase did not change much. Compared with the performance of the first week, there was a slight increase in the second and fourth weeks and a slight decrease in the third week. Compared with the first week, the average 


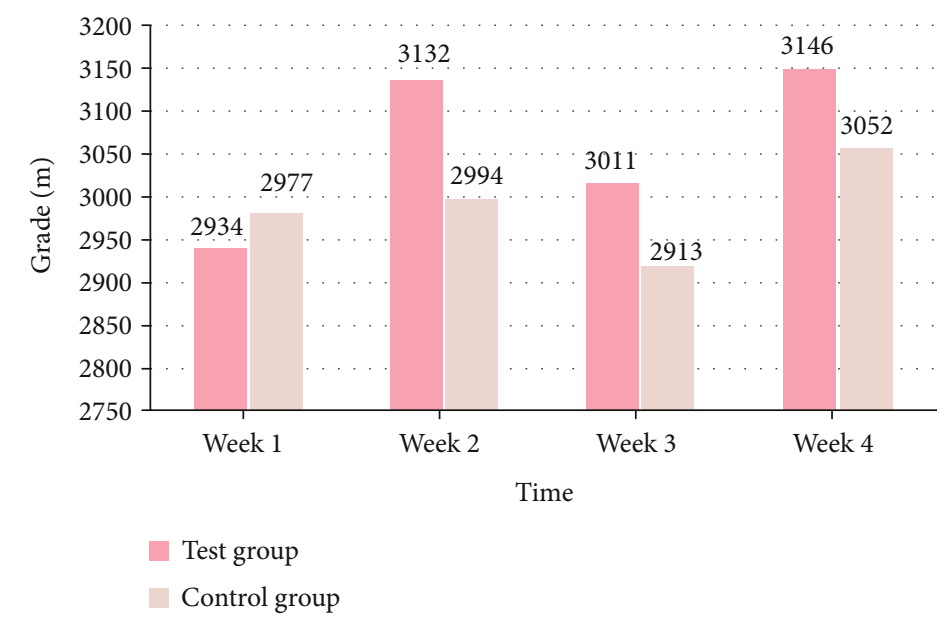

FIGURE 5: Results of the experimental group and the control group.

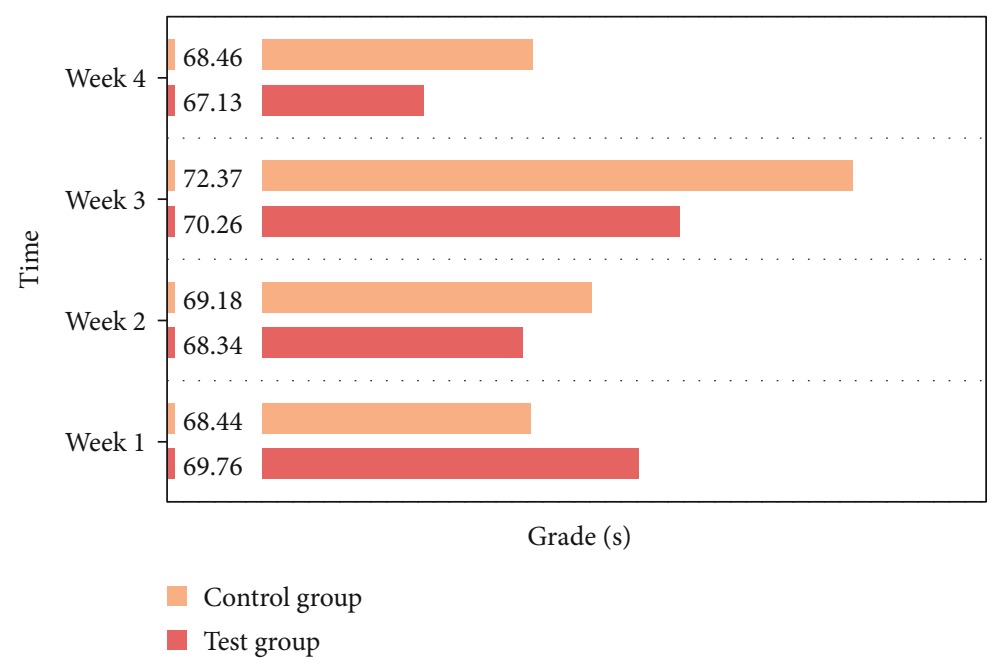

FIGURE 6: Results of the experimental group and the control group.

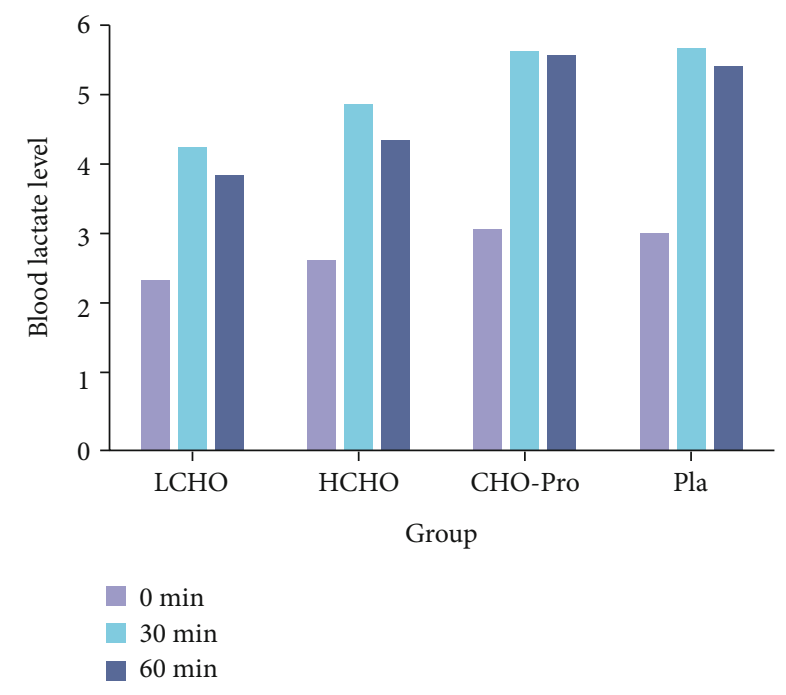

FIGURE 7: Changes in venous blood lactate levels. performance of the 400-meter race in the control group decreased slightly in the second week, significantly decreased in the third week (up to 7.69 seconds compared to the first week), and slightly increased in the fourth week. The average performance of the 1000-meter run in the experiment did not change much, and compared to the first week's performance in the second and third weeks, there was a slight improvement, and the fourth week's performance improved, while the control group was compared with the first week's 100 -run average performance in the second week.

As shown in Figure 7, there is no statistical difference in the venous blood lactic acid level in the body before fluid intake, and it is comparable; time has a significant difference in the effect of lactic acid level, and compared with each other, $30 \mathrm{~min}$ and $60 \mathrm{~min}$ are both significantly higher than the quiet state $(P<0.05)$.

\section{Conclusions}

The birth of mobile sensors is that people perceive the world more directly and maximize the functions of the existing 
network, so that human beings have a better understanding of the world. The century is the era of information, and mobile sensors will become one of the main challenges for people to research in the information field. Some countries have recognized its importance and set it as a difficult problem that must be overcome to occupy the information field. In this paper, based on mobile sensor network technology, the effect of sports nutrition supplement on physical growth is tested. In the exercise test in the afternoon of the exercise test in this study, there was no significant difference in exercise time when different fluid supplement programs were exhausted, indicating that the effects of different fluid supplement programs on exercise performance were basically the same. It is difficult for a single sports drink supplement to quickly show a very outstanding effect on the macroscopic exercise ability, and its promoting effect is often reflected in the change of specific biochemical indicators. By stabilizing the internal environment of the exercise body and replenishing the consumed energy materials, exercise time can be prolonged, exercise capacity can be improved, and recovery period can be shortened, but the manifestation of its effects may require long-term intervention. In this study, the Trp in group A after two weeks of exercise with nutritional supplements was significantly lower than that after exercise without nutritional supplements, which was earlier than three weeks in group B. In addition, the leucine and isoleucine levels of group A after three weeks of exercise with nutritional supplements were significantly higher than those of the same group before exercise without supplements. Although the indexes of group B increased correspondingly, there was no significant difference. These results and the subjective scale indicators indicate to a certain extent that the group A nutrition supplement with Gln is better than the group B nutrition supplement in improving the body's recovery speed.

\section{Data Availability}

The data that support the findings of this study are available from the corresponding author upon reasonable request.

\section{Conflicts of Interest}

The authors declared no potential conflicts of interest with respect to the research, authorship, and/or publication of this article.

\section{References}

[1] C. Yang, C. Yang, Z. Yang, and Z. Deng, "Robust weighted state fusion Kalman estimators for networked systems with mixed uncertainties," Information Fusion, vol. 45, pp. 246265, 2019.

[2] H. Song and M. Brandt-Pearce, "A 2-D discrete-time model of physical impairments in wavelength-division multiplexing systems," Journal of Lightwave Technology, vol. 30, no. 5, pp. 713-726, 2012.

[3] M. Sousa, M. J. Fernandes, J. Soares, P. Moreira, and V. H. Teixeira, "Nutritional supplement-usage associated character- istics of high-performing athletes," British food journal, vol. 118, no. 1, pp. 26-39, 2016.

[4] F. C. Wardenaar, I. J. Ceelen, J. W. van Dijk et al., "Nutritional supplement use by Dutch elite and sub-elite athletes: does receiving dietary counseling make a difference?," International Journal of Sport Nutrition and Exercise Metabolism, vol. 27, no. 1, pp. 32-42, 2017.

[5] S. Heaney, H. O'Connor, S. Michael, J. Gifford, and G. Naughton, "Effectiveness of education interventions designed to improve nutrition knowledge in athletes: a systematic review," Sports Medicine, vol. 49, no. 3, pp. 1769-1786, 2019.

[6] F. Gao, G. Wang, L. Wang, and N. Guo, "Phytosterol nutritional supplement improves pregnancy and neonatal complications of gestational diabetes mellitus in a double-blind and placebo-controlled clinical study," Food \& Function, vol. 8, no. 1, pp. 424-428, 2017.

[7] H. Imamura, K. Nishikawa, K. Kishi et al., "Effects of an oral elemental nutritional supplement on post-gastrectomy body weight loss in gastric cancer patients: a randomized controlled clinical trial," Annals of Surgical Oncology, vol. 23, no. 9, pp. 2928-2935, 2016.

[8] M. Paulo, "Glutamine in critically ill patients: is it a fundamental nutritional supplement?," Revista Brasileira de terapia intensiva, vol. 28, no. 2, pp. 100-103, 2016.

[9] O. Theou, I. Chapman, L. Wijeyaratne et al., "Can an intervention with testosterone and nutritional supplement improve the frailty level of under-nourished older people?," journal of Frailty \& Aging, vol. 5, no. 4, pp. 247-252, 2016.

[10] D. N. Pduraru, "The use of nutritional supplement in Romanian patients-attitudes and beliefs," Farmácia, vol. 67, no. 6, pp. 1060-1065, 2019.

[11] Y. Feng, "Functionality of dairy proteins and vegetable proteins in nutritional supplement powders: a review," International Food Research Journal, vol. 26, no. 6, pp. 1651-1664, 2020.

[12] B. Baker, B. Probert, D. Pomeroy, J. Carins, and K. Tooley, "Prevalence and predictors of dietary and nutritional supplement use in the Australian army: a cross-sectional survey," Nutrients, vol. 11, no. 7, p. 1462, 2019.

[13] H. Essa, "Nutritional supplement prepared from whole meal wheat flour, soya bean flour, flaxseed and anise seeds for alleviating the menopausal symptoms," Pakistan Journal of Science, vol. 18, no. 7, pp. 381-388, 2018.

[14] E. Ayayee and E. Chivandi, "Quercus Robur (English oak) seed: a potential energy, oleic and cis-linoleic acid rich nutritional supplement in South Africa," Pakistan Journal of Botany, vol. 50, no. 5, pp. 1907-1912, 2018. 